THERYA, diciembre, 2011

Vol.2(3):279-283

DOI: 10.12933/therya-11-41

\title{
Nuevos registros de Sylvilagus floridanus en la Península de Yucatán, México
}

\author{
Enrique Escobedo-Cabrera ${ }^{1}$ y Consuelo Lorenzo ${ }^{2}$
}

\section{Abstract}

We document current records of the eastern cottontail rabbit, Sylvilagus floridanus, in the Yucatan Peninsula with the goal of improving the available information with regards to their distribution in Mexico. The presence of specimens of S. floridanus collected, observed and by tracks (pellets) extends the distribution of this species in the Yucatan Peninsula approximately $145 \mathrm{~km}$ to the northeast of Champotón, Quintana Roo, and $205 \mathrm{~km}$ to the southwest of Laguna Chichankanab, Quintana Roo, the nearest localities registered previously. Monitoring efforts are needed so that the conservation status of the populations of this species in the Yucatan Peninsula can be determined.

Keywords: Eastern cottontail rabbit, conservation, distribution, Mexico, Yucatan Peninsula.

\section{Resumen}

Presentamos registros actuales del conejo castellano, Sylvilagus floridanus, en la Península de Yucatán con el fin de ampliar el conocimiento de su distribución en México. La presencia de ejemplares de $S$. floridanus colectados, observados y por rastros (excretas) amplía la distribución de la especie en la Península de Yucatán aproximadamente 145 km al noreste de Champotón, Campeche y 205 km al suroeste de Laguna Chichankanab, Quintana Roo, las localidades más cercanas registradas previamente. Es necesario monitorear las poblaciones de esta especie en la Península de Yucatán para estimar su estado de conservación.

Palabras clave: Conejo castellano, conservación, distribución, México, Península de Yucatán.

\section{Introducción}

El conejo castellano, Sylvilagus floridanus presenta la distribución geográfica más amplia del género Sylvilagus de Norteamérica. Abarca desde el sureste de Canadá, gran parte de México hasta el noroeste de Sudamérica (Diersing 1978; Chapman et al. 1980; Hall

\footnotetext{
${ }^{1}$ Av. Centenario Km 5.5, carretera Chetumal-Calderitas, Chetumal, Quintana Roo, CP 77014, E-mail: escobedo@ecosur. $\mathrm{mx}(\mathrm{EEC})$

${ }^{2}$ Departamento Ecología y Sistemática Terrestres, El Colegio de la Frontera Sur. Carretera Panamericana y Periférico Sur s/n, San Cristóbal de Las Casas, Chiapas, CP 29290, E-mail: clorenzo@ecosur.mx (CL)
} 
1981). Sylvilagus floridanus coexiste a lo largo de su distribución en México con tres especies más de Sylvilagus (S. audubonii, S. brasiliensis, S. cunicularius), y con cuatro especies de Lepus (L. alleni, L. californicus, L. callotis, L. flavigularis; Chapman y Ceballos 1990). Habita en valles, planicies, montañas con bosques de coníferas y de encinos, bosques tropicales, pastizales y matorrales xerófilos (Chapman y Ceballos 1990; Nelson 1904, 1909), desde el nivel del mar hasta aproximadamente 3,200 msnm (Chapman et al. 1980). Esta especie presenta pelaje dorsal largo y denso color canela rojizo, el color del centro del dorso es gris claro a negro; el vientre y la parte ventral de la cola son blancos a blancos crema (Hall 1981; Ceballos y Galindo 1984; Chapman et al. 1982), y presenta un parche amarillo-café en la nuca (Chapman et al. 1980). El cuello y la parte ventral de las patas son de color canela rojizo, mientras que la parte dorsal de las patas tiende a ser café claro (Álvarez-Castañeda 1996).

La distribución geográfica de las diez subespecies de $S$. floridanus con distribución en México ha sido pobremente documentada (Cervantes et al. 1997; Lorenzo y Cervantes 2005). Los registros de esta especie son escasos en algunos estados de la República Mexicana, como los pertenecientes a la Península de Yucatán. En la región occidental de la península de Yucatán se distribuye $S$. f. yucatanicus, con pocos registros, que incluyen Champotón en Campeche, Progreso (localidad tipo) y Chichen-Itzá en Yucatán (Jones et al. 1974), y Laguna Chichankanab en Quintana Roo (Hall 1981; Cervantes et al. 1997; Lorenzo y Cervantes 2005). Otros registros adicionales son: Campeche: Campeche; Yucatán: Calotmul, Izamel, Mérida, Progreso, Ria Lagartos, San Anselmo, San Felipe, Silam, Temax, Tzalam, Uxmal, Xbac, Yalahau y Yaxcach (Jones et al. 1974). No existen registros publicados de alguna otra especie de Sylvilagus en la Península de Yucatán; sin embargo, S. brasiliensis, especie geográficamente más cercana a S. floridanus estuvo presente a fines del Pleistoceno en la Península de Yucatán y aunque su distribución está pobremente documentada en la península, podría encontrarse en la porción sur de la misma (Arroyo-Cabrales y Álvarez 2003; Jones et al. 1974).

La finalidad de este reporte es ampliar el conocimiento de la distribución geográfica de S. floridanus en la península de Yucatán, México.

Un macho juvenil de S. floridanus (ECOSUR ECOCHM 1861) fue colectado en Dzibalchén, Municipio de Hopelchén, Campeche (19.4525 N, 89.758333 O), en acahual de selva mediana subperenifolia, el 8 de abril de 1999. Otro ejemplar, una hembra juvenil de $S$. floridanus (ECOSUR ECOCHM 2556) fue colectado a $1.57 \mathrm{~km}$ sureste de Bulukax, Quintana Roo (19.951019 N, 88.760869 O), en pastizal el 31 de mayo de 2011. Ambos ejemplares están depositados en la Colección de Mamíferos del Museo de Zoología de El Colegio de la Frontera Sur en la unidad Chetumal. La presencia del primer ejemplar amplía la distribución de la especie en la Península de Yucatán aproximadamente 102 km al noreste de Champotón, Campeche y 112 km al suroeste de Laguna Chichankanab, Quintana Roo, las localidades más cercanas registradas previamente.

Las medidas somáticas del macho y la hembra juveniles son (en $\mathrm{mm}$ ), respectivamente: longitud total, 470, 410; longitud de la cola, 43, 55; pata trasera, 95, 96; oreja desde la escotadura, 69, 66; peso, 1,600 gramos (para ambos). La morfología de estos ejemplares concuerda en general con las características diagnósticas de la especie, mencionadas previamente.

Algunas medidas craneales del machoy hembra juveniles son (en $\mathrm{mm}$ ), respectivamente: 


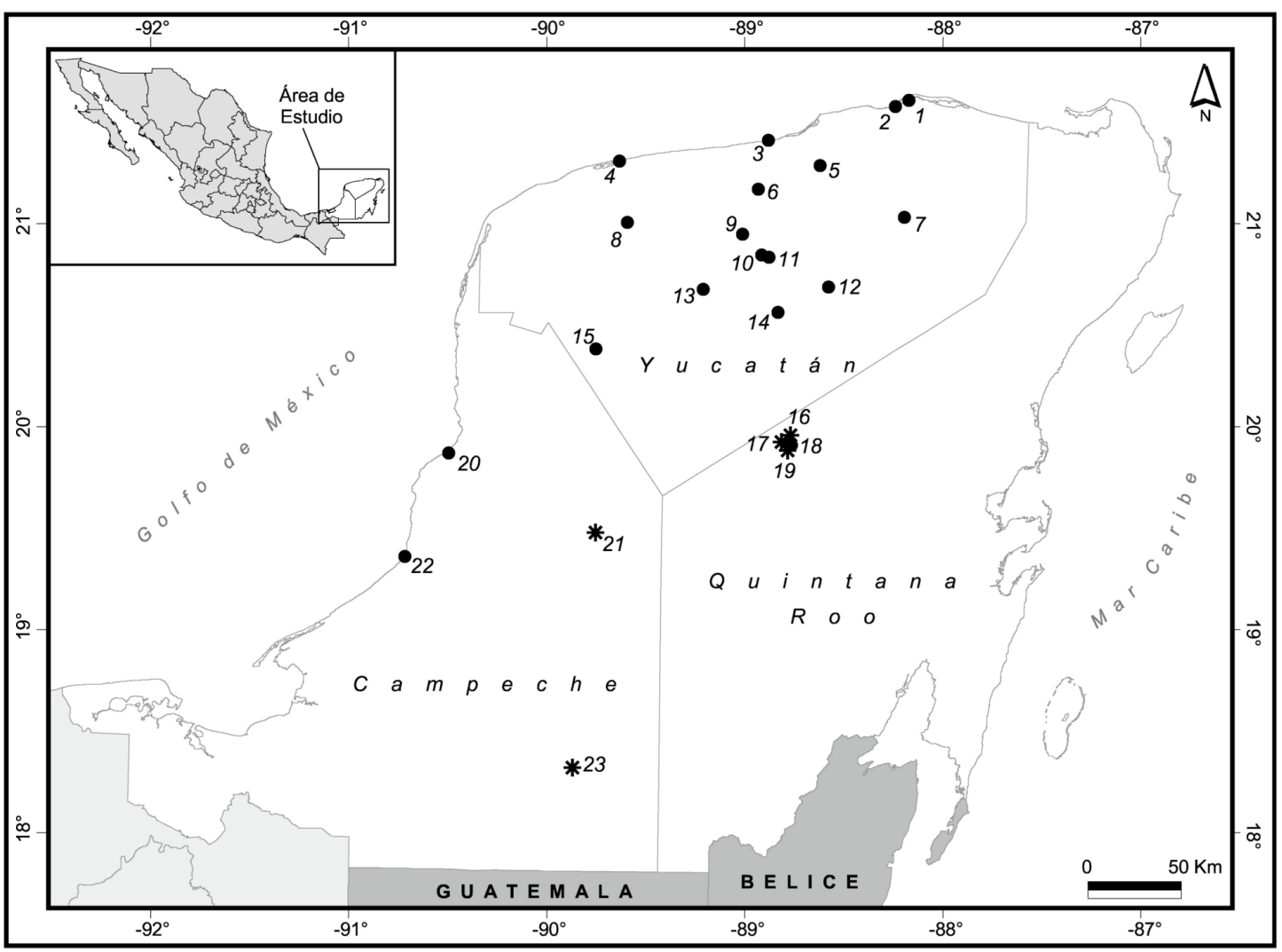

Figura 1. Registros actuales (asteriscos) y previos (puntos) del conejo castellano, Sylvilagus floridanus yucatanicus en la Península de Yucatán, México. 1 = Ria Lagartos; 2 =San Felipe; $3=$ Silam (Dzilam): $4=$ Progreso; $5=$ San Anselmo; $6=$ Temax; 7 = Calotmul, 8 = Mérida; $9=$ Izamel; $10=$ Yaxcach; $11=$ Tzalam; $12=$ Chichen Itza; $13=$ Yalahau; $14=$ Xbac; 15 = Uxmal; $16=1.5 \mathrm{~km}$ este Bulukax; $17=1.5 \mathrm{~km}$ norte Dziubché; 18 = Rancho la Ceiba; $19=4.3 \mathrm{~km}$ sureste Dziubché; 20 = Campeche; 21 = Dzibalchén; 22 = Champotón; 23 = Calakmul (Jones et al. 1974; Hall 1981; Cervantes et al. 1997; Lorenzo y Cervantes 2005).

anchura de la caja craneana, 26.2, 25.0; altura del cráneo, 30.0, 31.0; longitud de la mandíbula, 36.0, 39.4; longitud de dientes del maxilar, 14.2, 13.3; longitud de dientes de la mandíbula, 15.2, 14.6; longitud del diastema, 20.4, 20.5; longitud de los incisivos; ,- 8 ; longitud de los nasales, 34.0, 32.2.

Adicionalmente, se observaron dos ejemplares de esta especie, el primero el 8 de julio de 2010 en la Reserva de la Biosfera Calakmul km 27, en la Aguada El Ramonal (18.316139 N, -89.856556 O), Campeche, en selva alta; y el segundo el 23 de abril de 2011 a 1.5 km al norte de Dziuché (19.9175 N, -88.807222 O), Quintana Roo, en selva mediana subperennifolia. La presencia del primer ejemplar observado amplía la distribución de la especie en la Península de Yucatán aproximadamente $145 \mathrm{~km}$ al sureste de Champotón, Campeche y 205 km al suroeste de Laguna Chichankanab, Quintana Roo, las localidades más cercanas registradas previamente.

Las diferencias morfológicas existentes entre S. floridanus y S. brasiliensis son notorias, lo que hace fácil su identificación en campo, aunque llegaran a coexistir en la parte sur de la Península de Yucatán; por ejemplo, en la Reserva de la Biosfera Calakmul. A diferencia de $S$. floridanus, S. brasiliensis es un conejo de tamaño pequeño dentro del género Sylvilagus, su dorso es de color pardo obscuro, el vientre es más claro aunque en la región de la garganta es también obscuro, al igual que la parte dorsal de la cola y a diferencia de otras especies de Sylvilagus (con excepción de S. insonus), la parte ventral es del mismo color que el dorso del cuerpo (Diersing 1981; Hall 1981). 
También se recolectaron excretas de S. floridanus el 8 de diciembre de 1992 a $4.3 \mathrm{~km}$ al sureste de Dziuché (19.877236 N, -88.773839 O), a orillas de la Laguna Chichancanab, Quintana Roo, en pastizal. Las características de las excretas de $S$. floridanus recolectadas concuerdan con las reportadas en literatura (Aranda 2000), ya que son de color pardo, de forma irregular y un diámetro máximo alrededor de $1 \mathrm{~cm}$. El registro de estas excretas refuerza la presencia de $S$. floridanus en Chichancanab, localidad mencionada previamente en la literatura

Es necesario explorar nuevas áreas en la Península de Yucatán para definir con mayor precisión la distribución de $S$. floridanus y determinar si en la actualidad existe $S$. brasiliensis en la misma. Se requiere también llevar a cabo monitoreos de $S$. floridanus para incrementar su conocimiento y estado de conservación.

Agradecimientos

Agradecemos a H. Weissenberger por la elaboración del mapa y al Sr. S. Yam del ejido Dziuché, Quintana Roo y a J. Bolaños por su apoyo en el campo.

Referencias

Átvarez-Castañeda, S. T. 1996. Los mamíferos del estado de Morelos. Centro de Investigaciones Biológicas del Noroeste, S. C. La Paz, Baja California Sur, México.

Aranda, M. 2000. Huellas y otros rastros de los mamíferos grandes y medianos de México. Comisión Nacional para el Conocimiento y Uso de la Biodiversidad, Instituto de Ecología, A.C. Xalapa, Veracruz, México. 212 pp.

Arroyo-Cabrales, J., y T. Álvarez. 2003. Chapter 10. A preliminary report of the Late Quaternary Mammal Fauna from Loltún Cave, Yucatán, Mexico. Pp. 262-272 in Ice Age cave faunas of North America (Schubert, B. W. et al., eds.). Indiana University Press and Denver Museum of Nature and Science.

Ceballos, G., y C. Galindo. 1984. Mamíferos silvestres de la Cuenca de México. Limusa, Distrito Federal, México.

Cervantes, F. A., C. Lorenzo, y M. D. Engstrom. 1997. New records of the Eastern cottontail (Sylvilagus floridanus) and black-tailed jackrabbit (Lepus californicus) in Mexico. The Texas Journal of Science 49:75-77.

Chapman, J. A., J. G. Hockman, y C. M. M. Ojeda. 1980. Sylvilagus floridanus. Mammalian Species 136:1-8.

Chapman, J. A., J. G. Hockman, y W. R. Edwards. 1982. Cottontails. Pp. 83-123 in Wild Mammals of North America (Chapman, J. A., y G. A. Feldhamer, eds.). The Johns Hopkins University Press. Baltimore and London.

Chapman, J. A., y G. Ceballos. 1990. The Cottontails. Pp. 95-110 in Rabbits, hares and pikas, Status survey and Conservation Action plan (Chapman, J. A., y J. E. C. Flux, eds.). International Union for the Conservation of Nature and Natural Resources, Gland, Switzerland.

Diersing, V. E. 1978. A systematic revision of several species of cottontails (Sylvilagus) from North and South America. Tesis Doctoral. University of Illinois at UrbanaChamaign, Illinois.

Diersing, V. E. 1981. Systematic status of Sylvilagus brasiliensis and S. insonus from North 
America. Journal of Mammalogy 62:539-556.

Hall, E. R. 1981. The mammals of North America. Second ed. John Wiley and Sons. New York.

Jones, J. K. JR., H. H. Genoways, y J. D. Smith. 1974. Annotated checklist of mammals of the Yucatan Peninsula, Mexico. III. Marsupialia, Insectivora, Primates, Edentata, Lagomorpha. Occasional Papers The Museum Texas Tech University 23:1-12.

Lorenzo, C., y F. A. Cervantes. 2005. El conejo Sylvilagus floridanus (J. A. Allen, 1890). Pp. 843-845 in Los Mamíferos Silvestres de México (Ceballos, G., y G. Oliva, eds.). CONABIO y Fondo de Cultura Económica. México, Distrito Federal.

Netson, E. W. 1904. Description of seven new rabbits from Mexico. Proceedings of the Biological Society of Washington 17:103-110.

Nelson, E. W. 1909. The rabbits of North America. North American Fauna 29:1-314.

Sometido: 12 de julio 2011

Revisado: 21 de octubre 2011

Aceptado: 28 de noviembre 2011

Editor asociado: Jesús Maldonado

Diseño gráfico editorial: Gerardo Hernández 